\title{
How to cage an egg
}

\author{
Oded Schramm \\ University of California, San Diego, Department of Mathematics, La Jolla, CA 92093, USA \\ Oblatum 18-V-1991
}

Summary. This paper proves that given a convex polyhedron $P \subset \mathbb{R}^{3}$ and a smooth strictly convex body $K \subset \mathbb{R}^{3}$, there is some convex polyhedron $Q$ combinatorically equivalent to $P$ which midscribes $K$; that is, all the edges of $Q$ are tangent to $K$. Furthermore, with some stronger smoothness conditions on $\partial K$, the space of all such $Q$ is a six dimensional differentiable manifold.

\section{Introduction}

For any $n=3,4,5, \ldots$ one can find a convex $n$-gon in the plane so that all its vertices lie on the unit circle. In fact, the convex hull of any $n$ distinct points on the unit circle gives such an $n$-gon. The situation in 3-space is rather different. Steinitz [St] has shown that there are convex polyhedra $P \subset \mathbb{R}^{3}$ so that there is no combinatorically equivalent polyhedron having all the vertices on the unit sphere. By duality, it follows that there are combinatorial types of convex polyhedra that are not realizable with all the (2-dimensional) faces tangent to the unit sphere.

Schulte $[\mathrm{Schu}]$ has generalized the above result of Steinitz, by showing that for pairs of integers $(m, d)$ satisfying $0 \leqq m<d, d>2,(m, d) \neq(1,3)$ there are combinatorial types of $d$-dimensional polytopes which are not $(m, d)$-scribable; that is, they cannot be realized in $d$-space in such a way that all the $m$-dimensional faces are tangent to the unit $(d-1)$-sphere. The case $(m, d)=(1,3)$, is an exception. Koebe [Koe] has claimed that every 3 dimensional convex polyhedron is combinatorically equivalent to a polyhedron which midscribes the unit sphere; that is, all its edges are tangent to the unit sphere. However, the proof in [Koe] is only for polyhedra which are simple or simplicial. The result for general polyhedra follows from Andreev's Theorem [An1, An 2], as Thurston [Th, Chap. 13] observed. The midscribing polyhedron is unique, up to projective transformations which preserve the sphere.

Schulte also introduced the question of replacing the Euclidean sphere in the above by other convex bodies. It is the purpose of this paper to generalize the Koebe-Andreev-Thurston result in this direction by proving: 
1.1 Midscribability Theorem. Let $P$ be a (3-dimensional) convex polyhedron, and let $K \subset \mathbb{R}^{3}$ be a smooth strictly convex body. Then there exists a convex polyhedron $Q \subset \mathbb{R}^{3}$ combinatorially equivalent to $P$ which midscribes $K$.

With stronger smoothness conditions on $K$, one can say more:

1.2 Theorem. In Theorem 1.1, if for some integer $k \geqq 1 \partial K$ is $C^{k+1}$-smooth and has positive Gaussian curvature everywhere, then the space of such midscribing $Q$ is a six dimension $C^{k}$-smooth manifold.

This also implies that the space of midscribing $Q$ is $C^{\infty}$, if $\partial K$ is $C^{\infty}$ and has positive Gaussian curvature.

A few words about the proof of Theorem 1.1. The main part of the proof is establishing the theorem for the case where $\partial K$ is $C^{2}$-smooth and has positive Gaussian curvature everywhere, and then the general case follows by approximations and convergence. We consider the space of all configurations; where a configuration is, by definition, an indexed collection of planes and points in $\mathbb{R}^{3}$; the points being indexed by the vertices of $P$, and the planes are indexed by the faces of $P$. A configuration corrresponds to a $P$-type $K$-midscribing polyhedron if the obvious conditions hold: the point corresponding to any vertex $v$ of $P$ belongs to every plane corresponding to a face of $P$ containing the vertex $v$, the line segment joining the points corresponding to two vertices which share an edge in $P$ is tangent to $K$, etc. With the presence of the stronger smoothness assumptions on $K$, these different conditions determine differentiable submanifolds of configuration space, and so, each point in the intersection of these submanifolds corresponds to a $P$-type $K$-midscribing polyhedron. We use geometric observations and a combinatorial argument to show that any intersection of these submanifolds is transverse. Transversality then implies that if we have a $P$-type $K$-midscribing polyhedron $Q$, then for a sufficiently small perturbation $K^{\prime}$ of $K$ a $K^{\prime}$ midscribing $P$-type polyhedron $Q^{\prime}$ can be found. This enables us to transport midscribing polyhedra from one $K$ to another, and the proof is then easily completed.

Certainly, the central part of this argument is the proof of transversality. The method in which we establish transversality is in many respects reminiscent of Cauchy's approach to rigidity of polyhedra ([Ca, Ro]). In a forthcoming paper the author intends to elaborate on this matter, and to use some of the techniques presented here to obtain new proofs of infinitesimal Cauchy rigidity and related results.

As noted above, Thurston's proof that the sphere can be midscribed by a polytope of given combinatorics is an application of Andreev's Theorem. As Thurston observed, Andreev's Theorem can be reinterpreted to obtain the Circle Packing Theorem, which says that any planar graph can be realized as the tangency graph of a circle packing on the sphere. In [Schr] the Circle Packing Theorem is generalized to packings of shapes other than circles. Using this generalization, Theorem 1.1 is proved there in the case that $P$ is a simple or a simplicial polyhedron. Although this may not be apparent to the reader, the techniques of this paper are also closely related to the theory of packings in two dimensions. In fact, much of the arguments here were conceived as part of a differentiable proof giving uniqueness and existence of packings, in the spirit of [Schr]. Later, the method of [Schr] superseded the differentiable proof. Fortunately, this differentiable approach is most useful for the purposes of this work. 
It is worthwhile to note that Thurston's route from circle packings to spheremidscribing polyhedra can be reversed, and so we obtain a new proof of the Circle Packing Theorem as an additional reward for our efforts. (See 6.1.)

Acknowledgements. I would like to express thanks to Egon Shulte for suggesting to try and use my packing theorems to obtain polyhedra that midscribe given convex bodies. I am also very grateful to Bill Thurston, who advised me to try the differentiable approach to obtain a uniqueness result for packings.

\section{Preliminaries}

The main purpose of this section is to define some terms we later use, and to introduce notations. We freely use some of the most elementary notions in convexity (good sources for these are, for example, $[\mathrm{Eg}, \mathrm{Gr}]$ ), and these are defined here only if some confusion is anticipated. Similarly, we assume some very rudimentary knowledge of differential topology, especially transversality of submanifolds.

A convex body in $\mathbb{R}^{3}$ is a compact convex subset of $\mathbb{R}^{3}$ which has interior points. A smooth convex body is a convex body which has a unique supporting plane at every boundary point (this is equivalent to the boundary being $C^{1}$-smooth), and a strictly convex body, is a convex body whose boundary does not contain any nontrivial line segment.

Given points $x, y \in \mathbb{R}^{n}$, we use the notation $[x, y]$ to denote the line segment joining $x$ and $y$.

A polyhedron in $\mathbb{R}^{3}$ is the convex hull of finitely many points in $\mathbb{R}^{3}$ which are not all coplanar. Let $P$ be some arbitrary polyhedron in $\mathbb{R}^{3}$, which will be fixed henceforth. We denote the set of vertices of $P$ by $V$, the set of edges by $E$, and the set of (2-dimensional) faces by $F$. A $P$-type polyhedron will mean a polyhedron which is combinatorically equivalent to $P$.

The extended graph of $P, G^{+}=G^{+}(P)$, is the graph whose vertex set is $V \cup F$, and whose edges are $E \cup V F$, where $V F$ denotes the collection of all pairs $\langle v, f\rangle$ so that $v \in V, f \in F$ and the vertex $v$ belongs to the face $f$. A vertex $i$ in the graph $G^{+}$will be called a $V$-vertex if $i \in V$, or an $F$-vertex if $i \in F$. Hopefully, the fact that a face of $P$ is a vertex of $G^{+}$will cause no confusion. An edge $\langle i, j\rangle$ of $G^{+}$is a V-V edge if $\langle i, j\rangle \in E$, and is termed a V-F edge if $\langle i, j\rangle \in V F$.

$G^{+}$has an embedding in $S^{2}$, canonical up to homeomorphism, which can be visualized on the boundary of $P$ by choosing an interior point in every face and connecting it to the vertices of that face. We will always consider $G^{+}$with this embedding, which determines a triangulation of $S^{2}$. As above, if $i, j$ are vertices of $G^{+}$which are joined by an edge, then $\langle i, j\rangle$ will denote that edge. Similarly, the notation $\langle i, j, k\rangle$ will be used for a triangle of $G^{+}$; that is, a triangle in the triangulation induced by $\mathrm{G}^{+}$.

A polyhedron $Q \subset \mathbb{R}^{3}$ is said to midscribe a smooth strictly convex body $K \subset \mathbb{R}^{3}$ if every edge of $Q$ is tangent to $K$ (that is, to $\partial K$ ).

2.1 Observations. Let $K \subset \mathbb{R}^{3}$ be a smooth and strictly convex body, and let $Q$ be a K-midscribing polyhedron.

(1) If $m$ is a plane containing a face of $Q$, then the polygon $m \cap Q$ circumscribes the smooth convex set $m \cap K$ in the plane $m$. 
(2) If $v, u$ are vertices of $Q$, then the line segment $[v, u]$ intersects $K$.

Proof. (1) is obvious. By definition, (2) holds if $\langle v, u\rangle$ is an edge of $Q$. Otherwise, let $E_{v}$ be the set of edges of $Q$ incident with $v$. Each edge $\langle v, w\rangle \in E_{v}$ contains some point $p_{\langle v, w\rangle} \in K$. But $[v, u]$ intersects the convex hull of the points $p_{e}$, $e \in E_{v}$, and $K$ is convex. Therefore (2) holds.

\section{Configuration space and its submanifolds}

In this section $K$ will denote an arbitrary smooth strictly convex body in $\mathbb{R}^{3}$.

Let $Z$ be the space $\left(\mathbb{R}^{3}\right)^{V} \times\left(G r_{2}\right)^{F}$, where $G r_{2}$ is the space of all oriented affine planes in $\mathbb{R}^{3}$. In other words, a point $z \in Z$ gives a choice of a point in $\mathbb{R}^{3}$ for each vertex $v \in V$ of $P$, and a choice of an oriented plane for each face $f \in F$. $Z$ will be called the configuration space and a point $z \in Z$ will be called a configuration. For a configuration $z \in Z$ we denote by $z_{v}$ the point in $\mathbb{R}^{3}$ corresponding to a vertex $v \in V$, and similarly, $z_{f}$ will denote the plane corresponding to a face $f \in F$.

For each V-F edge $\langle v, f\rangle \in V F$, let $S_{\langle v, f\rangle}$ be the submanifold of $Z$ consisting of the configurations $z \in Z$ for which $z_{v}$ is contained in the plane $z_{f} . S_{\langle v, f\rangle}$ is a $C^{\infty}$-smooth submanifold of $Z$ having codimension 1 .

Let $Z_{+}=Z_{+}(K)$ be the set of configurations $z \in Z$ which avoid $K$ in the sense that $z_{v} \notin K$ for each $v \in V$. $Z_{+}$is an open set in $Z$.

Let $Z_{c}$ be the set of configurations $z$ so that for each face $f \in F$ all the points $z_{v}$ corresponding to vertices $v$ which do not belong to the face $f$ are below the plane $z_{f}$. Because the plane $z_{f} \in G r_{2}$ is oriented, it is meaningful to refer to the open half space above $z_{f}$, and to the one below $z_{f} . Z_{c}$ is also open in $Z$.

For each V-V edge $\langle v, u\rangle$ of $P$, let $S_{\langle v, u\rangle}=S_{\langle v, u\rangle}(K)$ be the set of all configurations $z \in Z_{+}$so that (the relative interior of) the line segment joining $z_{v}$ and $z_{u}$ is tangent to $\partial K$.

3.1 Lemma. If $\partial K$ is $C^{k+1}$-smooth (for some integer $k \geqslant 1$ ), and has positive Gaussian curvature everywhere, then $S_{\langle v, u\rangle}$ is a codimension $1 C^{k}$-smooth submanifold of $Z_{+}$.

The proof will be sketchy, because it has little relevance to our interests.

Proof. Let $\psi: \mathbb{R}^{2} \rightarrow \partial K$ be a $C^{k+1}$-smooth coordinate chart for $\partial K$. Define a mapping

by

$$
\tau: \mathbb{R}^{2} \times\left(\mathbb{R}^{2}-\{0\}\right) \times \mathbb{R}_{+} \rightarrow\left(\mathbb{R}^{3}\right)^{\{v, u\}}
$$

$$
\Psi(a, b, \lambda)_{v}=\psi(a)+d \psi_{a}(b), \quad \tau(a, b, \lambda)_{u}=\psi(a)-\lambda d \psi_{a}(b) .
$$

(We identify $T_{a} \mathbb{R}^{2}$ with $\mathbb{R}^{2}$, and consider $T_{\psi(a)} \partial K$ as a linear plane in $\mathbb{R}^{3}$.) Then the segment $\left[\psi(a, b, \lambda)_{u}, \tau(a, b, \lambda)_{v}\right]$ is tangent to $\partial K$ at $\psi(a)$. Because $\psi$ is $C^{k+1}$-smooth, it follows that $\widetilde{\psi}$ is $C^{k}$-smooth.

From the assumption that $\partial K$ is positively curved it is not hard to (geometrically) deduce that $d \tilde{\psi}$ is everywhere nonsingular. It then follows that $\tilde{\psi} \times$ id can be used for a coordinate chart for $S_{\langle v, u\rangle}$, where id is the identity map on $\left(\mathbb{R}^{3}\right)^{V-\{v, u\}} \times\left(G r_{2}\right)^{F}$. The lemma follows. 
Suppose that $Q$ is a $P$-type polyhedron in $\mathbb{R}^{3}$, and consider a particular combinatorial equivalence between $Q$ and $P$. Then there is a configuration $z$ corresponding to $Q$ : for each $v \in V, z_{v} \in \mathbb{R}^{3}$ is the position of the vertex corresponding to $v$ in $Q$, and for each $f \in F, z_{f}$ is the plane containing the face corresponding to $f$ in $Q$, oriented so that all the points $z_{v}$ with $v$ a vertex not in $f$ are below $z_{f}$. Clearly, this configuration $z$ will be in

$$
Z_{P} \stackrel{\text { def }}{=} Z_{c} \cap\left(\cap_{\langle v, f\rangle \in V F} S_{\langle v, f\rangle}\right) .
$$

Conversely, a configuration contained in $Z_{P}$ corresponds to a polyhedron $Q$ of combinatorial type $P$. We will say that such a configuration realizes $P$, or that $z$ is a realization of $P$, and $P(z)$ will denote the actual polyhedron in $\mathbb{R}^{3}$ which corresponds to $z$.

If a $P$-type polyhedron $Q$ actually midscribes $K$, then the corresponding configuration $z$ will be in

$$
Z_{P, K} \stackrel{\text { def }}{=} Z_{P} \cap Z_{+} \cap\left(\cap_{\langle v, u\rangle \in E} S_{\langle v, u\rangle}\right) .
$$

Conversely, every $z \in Z_{P, K}$ is a realization of a $K$-midscribing $P$-type polyhedron. So $Z_{P, K}$ is the set we are really interested in. It is left to the reader to convince himseif that the above statements are obvious.

\section{Transversality}

The section is mostly devoted to the following theorem.

4.1 Transversality Theorem. Let $K \subset \mathbb{R}^{3}$ be a strictly convex body with $C^{2}$-smooth positively curved boundary. Fix some edge $\left\langle v_{0}, v_{1}\right\rangle \in E$ of $P$, and let $f_{2} \in F$ be one of the two faces containing this edge. Let $x_{0}$ and $x_{1}$ be two points in $\mathbb{R}^{3}-K$ so that the line segment joining them is tangent to $K$, and let $y_{2}$ be an oriented plane containing $x_{0}$ and $x_{1}$. Denote by $Z^{i}, i=0,1,2$, the set of configurations $z \in Z$ so that $z_{v_{0}}=x_{0}, z_{v_{1}}=x_{1}$, and $z_{f_{2}}=y_{2}$, respectively. Let $E^{\prime} \stackrel{\text { def }}{=} E-\left\{\left\langle v_{0}, v_{1}\right\rangle\right\}$, and let $V F^{\prime} \stackrel{\text { def }}{=} V F-\left\{\left\langle v_{0}, f_{2}\right\rangle,\left\langle v_{1}, f_{2}\right\rangle\right\}$. Then the intersection

$$
Z_{P, K}^{\prime} \stackrel{\text { def }}{=} Z_{+} \cap Z_{c} \cap Z^{0} \cap Z^{1} \cap Z^{2} \cap\left(\cap_{\langle i, j\rangle \in E^{\prime} \cup V F^{\prime}} S_{\langle i, j\rangle}\right)
$$

is transverse. $^{1}$

Note that $Z^{0} \cap Z^{1} \subset S_{\left\langle v_{0}, v_{1}\right\rangle}$, that $Z^{0} \cap Z^{2} \subset S_{\left\langle v_{0}, f_{2}\right\rangle}$, and that $Z^{1} \cap Z^{2} \subset S_{\left\langle v_{1}, f_{2}\right\rangle}$. Therefore $Z_{P, K}^{\prime}$ is contained in $Z_{P, K}$. In fact, the configurations $z \in Z_{P, K}^{\prime}$ are precisely the realizations of $P$-type polytopes which midscribe $K$ and satisfy

${ }^{1}$ Recall that the intersection $\cap_{n} M_{n}$ of differentiable submanifolds of a differentiable manifold $M$ is transverse if at every point $z$ in the intersection the codimension of the intersection of the tangent spaces $\cap_{n} T_{z} M_{n}$ is equal to the sum of the codimensions $\sum_{n} \operatorname{codim}\left(T_{z} M_{n}\right)$, where the tangent spaces $T_{z} M_{n}$ are naturally considered as subspaces of $T_{z} M$. In particular, if the intersection is empty, then it is transverse. 
the additional conditions $z_{v_{0}}=x_{0}, z_{v_{1}}=x_{1}$ and $z_{f_{2}}=y_{2}$. The proof of the theorem will be divided into several subsections.

4.2 Codimension count. $Z_{+}$and $Z_{c}$ are open in $Z$, and thus have codimension 0 . Each of $Z^{0}, Z^{1}, Z^{2}$ has codimension 3 in $Z$. $\left(\operatorname{dim}\left(G r_{2}\right)=3\right)$. Each of the $S_{\langle v, u\rangle}$ and the $S_{\langle v, f\rangle}$ have codimension 1 . So the sum of the codimensions for the intersection (4.1) is $3+3+3+\left|E^{\prime}\right|+\left|V F^{\prime}\right|$, which is equal to $6+|E|+|V F|$.

We will now show that $|V F|=2|E|$. Consider any $\mathrm{V}-\mathrm{F}$ edge $\langle v, f\rangle \in V F$. There are precisely two edges of $E$ which are contained in the face $f$ and have $v$ as one of their vertices. Conversely, for each edge $\langle w, u\rangle \in E$ there are four $\mathrm{V}$-F edges $\langle v, f\rangle \in V F$ so that $v$ is $w$ or $u$ and $f$ is a face containing $\langle w, u\rangle$. Thus we have exhibited a 4 to 2 correspondence between $V F$ and $E$. This establishes $|V F|=2|E|$.

Now we see that the sum of the codimensions is $6+3|E|$. By Euler's formula this is equal to $3|V|+3|F|=\operatorname{dim}(Z)$. Thus, in order to prove transversality, we must show that at each configuration $z$ contained in the intersection (4.1), the intersection of the tangent spaces

$$
T_{z} Z^{0} \cap T_{z} Z^{1} \cap T_{z} Z^{2} \cap\left(\cap_{\langle i, j\rangle \in E^{\prime} \cup V F^{\prime}} T_{z} S_{\langle i, j\rangle}\right)
$$

has dimension 0 ; i.e., contains only the zero vector. Here we think of $T_{z} Z^{0}$, the tangent space at $z$ of the submanifold $Z^{0}$ of $Z$, as being contained in $T_{z} Z$, the tangent space of $Z$ at $z$, and similarly for the other submanifolds of $Z$.

4.3 The labeling associated with a tangent vector. Assume that $z \in Z_{P, K}^{\prime}$, and let $t \in T_{z} Z$ be a tangent vector at $z$. In the following, we will associate with $t$ a labeling of the edges of $G^{+}$. Every edge of $G^{+}$will be labeled with two labels, one label near each of its two endpoints. The possible labels are $+, 0,-$. We will use the notation $l_{i, j}$ to denote the label on the edge $\langle i, j\rangle$ near the vertex $i$. For $v \in V$ and for $f \in F, t_{v} \in T_{z_{v}} \mathbb{R}^{3}$ and $t_{f} \in T_{z_{f}} G r_{2}$ will denote the components of $t$ corresponding to $v$ and $f$, respectively. (If $\pi_{v}: Z \rightarrow \mathbb{R}^{3}$ is the projection $\pi_{v}(z)=z_{v}$, then $t_{v} \stackrel{\text { def }}{=} d \pi_{v}(t)$, and similarly for $t_{f}$.)

Consider first an edge of type V-V, $\langle v, u\rangle \in E$. Because $z \in S_{\langle v, u\rangle}$, the line segment joining $z_{v}$ to $z_{u}$ is tangent to $K$. Therefore, since $K$ is smooth, it follows that there is a unique plane, say $m_{\langle v, u\rangle}$, which is tangent to $K$ and contains $z_{v}$ and $z_{u}$. We will refer to the open half space determined by $m_{\langle v, u\rangle}$ which is disjoint from $K$ as being above $m_{\langle v, u\rangle}$, and the other open half space determined by $m_{\langle v, u\rangle}$ as being below $m_{\langle v, u\rangle}$. With respect to $t_{v}$ we discriminate between three possibilities: $t_{v}$ either points above $m_{\langle v, u\rangle}$, points below $m_{\langle v, u\rangle}$, or is parallel to $m_{\langle v, u\rangle}$. It $t_{v}$ points above $m_{\langle v, u\rangle}$, we label the part of the edge $\langle v, u\rangle$ which is near $v$ with a + (i.e., we set $l_{v, u}=+$ ). If it points below, we place there a - , and if parallel, a 0 . Similarly, the vector $t_{u}$ determines the labeling of $\langle v, u\rangle$ near $u$, and so this procedure defines the labeling for all the $\mathrm{V}-\mathrm{V}$ edges.

Now consider any $\mathrm{V}-\mathrm{F}$ edge $\langle v, f\rangle$. In the case where $t_{v}$ points above $z_{f}$, we label $l_{v, f}=+$, if it points below $z_{f}$, we set $l_{v, f}=-$, and if parallel, $l_{v, f}=0$.

We define similarly the labeling of $\langle v, f\rangle$ near $f$. Informally, if every infinitesimal variation of $z_{f}$ in the direction $t_{f}$ gives a plane $y \in G r_{2}$ having $v$ below it, then we label with a - If every such variation gives a plane $y \in G r_{2}$ having $v$ above it, then we label $l_{f, v}=+$. Otherwise, a 0 .

In order to make this more precise, we need a convenient interpretation for $t_{f}$. Associate with each plane $y \in G r_{2}$ the unique affine function on $\mathbb{R}^{3}$ which 
is positive above $y$, negative below $y$, and whose gradient at every point has norm 1. Denote this function by $\phi(y)$. This defines an embedding $\phi$ of $G r_{2}$ into the vector space $A\left(\mathbb{R}^{3}, \mathbb{R}\right)$ of all affine functions on $\mathbb{R}^{3}$. In this way, to $t_{f}$ corresponds the tangent vector $d \phi\left(t_{f}\right) \in T_{\phi\left(z_{f}\right)} A\left(\mathbb{R}^{3}, \mathbb{R}\right)$. As $A\left(\mathbb{R}^{3}, \mathbb{R}\right)$ is a vector space, the tangent space $T_{\phi\left(Z_{f}\right)} A\left(\mathbb{R}^{3}, \mathbb{R}\right)$ is canonically isomorphic to $A\left(\mathbb{R}^{3}, \mathbb{R}\right)$, and thus we will regard $d \phi\left(t_{f}\right)$ as an affine function on $\mathbb{R}^{3}$. We set $l_{f, v}=+, 0,-$, depending on whether $d \phi\left(t_{f}\right)(v)>0, d \phi\left(t_{f}\right)(v)=0$, or $d \phi\left(t_{f}\right)(v)<0$, respectively.

Having completed the definition of the labeling associated with $t$, we now discuss its properties. We continue our assumptions that $z \in Z_{P, K}^{\prime}$, and that $t \in T_{z} Z$ is a tangent vector at $z$.

4.4 Lemma. Let $\langle v, u\rangle$ be any V-V type edge, and let $e, f \in F$ be the two faces containing this edge. Then $l_{v, u}$, the label of $\langle v, u\rangle$ near $v$, is 'between' $l_{v, f}$ and $l_{v, e}$. Saying that a label $l$ is between two labels means that if one of the two labels is ' 0 ', then $l$ is equal to the other label, and if both labels are equal, then $l$ is also equal to them. ( $l$ can be any of $+, 0,-$, if the two labels are + and - .)

Proof. There is clearly no loss of generality in assuming that $z_{v}=0$, and, for simplicity of notation, we will adopt this assumption. Let $w_{1}, w_{2} \in V$ be the neighbors of $v$ other than $u$ which belong to the faces $e, f$, respectively. We think of $t_{v}$ as a vector in $\mathbb{R}^{3}$. Because $z_{w_{1}}, z_{w_{2}}$ and $z_{u}$ are linearly independent, we can write

$$
t_{v}=-\lambda_{1} z_{w_{1}}-\lambda_{2} z_{w_{2}}+\lambda_{3} z_{u}
$$

Now, on $\left[z_{v}, z_{w_{1}}\right]$ there is some point of $K$, and this point must be below $m_{\langle v, u\rangle}$, because $K$ lies below $m_{\langle v, u\rangle}$, except for the unique point in $m_{\langle v, u\rangle} \cap K$. Since $z_{v} \in m_{\langle v, u\rangle}$, this implies that $z_{w_{1}}$ is below $m_{\langle v, u\rangle}$. On the other hand $z_{w_{1}}$ is on the plane $z_{e}$ and is below $z_{f}$. A similar argument shows that $z_{w_{2}}$ is on $z_{f}$ and below $z_{e}$ and $m_{\langle v, u\rangle}$. Noting that $z_{u}$ and $0=z_{v}$ are in $m_{\langle v, u\rangle} \cap z_{e} \cap z_{f}$, it follows from the above and from (4.3) that $l_{v, e}=\operatorname{sign}\left(\lambda_{2}\right)$, that $l_{v, f}=\operatorname{sign}\left(\lambda_{1}\right)$, and that $l_{v, u}=\operatorname{sign}\left(\lambda_{1}\right)$ if $\lambda_{2}=0$ or $\operatorname{sign}\left(\lambda_{1}\right)=\operatorname{sign}\left(\lambda_{2}\right)$, and that $l_{v, u}=\operatorname{sign}\left(\lambda_{2}\right)$ if $\lambda_{1}=0$. This shows that $l_{v, u}$ is between $l_{v, e}$ and $l_{v, f}$, and proves the lemma.

4.5 Definitions. Consider our graph $G^{+}$and the labeling of it induced by $t$, and let $i$ be some vertex of $G^{+}$(either a V-vertex, or an F-vertex). If there is some edge $e$ of $G^{+}$which is incident with $i$ and has a nonzero label near $i$, then $i$ will be called live. Otherwise, $i$ is termed dead. Now suppose that $\langle i, j, k\rangle$ is a triangle of $G^{+}$. If the two labels near $i$ on $\langle i, j\rangle$ and $\langle i, k\rangle$ are + and - (i.e., $\left\{l_{i, j}, l_{i, k}\right\}=\{+,-\}$ ), then we consider this as a sign change in the triangle $\langle i, j, k\rangle$ at the vertex $i$. If one of these labels is 0 , and the other is + or - , then we consider it as half a sign change. If both labels are 0 , then it is not considered a sign change if $i$ is dead, it is considered half a sign change if $i$ is a live $\mathrm{V}$-vertex, and it is considered a full sign change if $i$ is a live F-vertex. See Fig. 4.1.

The reason for the difference in the way V-and F-vertices are treated is that we have included $\mathrm{V}-\mathrm{V}$ edges in $\mathrm{G}^{+}$, but did not include F-F edges. The following lemma provides some motivation for these definitions. 


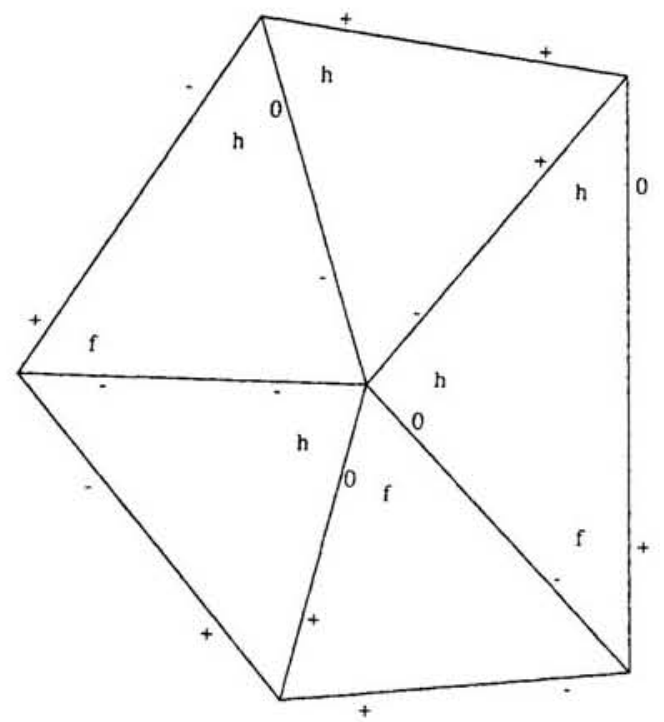

Fig. 4.1. An example of a portion of the labeled graph. The center vertex is an F-vertex, the others are V-vertices. A full sign change is marked by $f$, and half sign changes are marked $h$. (Non sign changes are unmarked)

4.6 Lemma (Sign changes around a vertex) Let $i$ be any V-vertex or F-vertex. (a) The total number of sign changes at $i$, counted according to the definition above, is at most 2. (b) $i$ is a live vertex if, and only if, $t_{i} \neq 0$.

Proof. Consider the case where $i$ is an F-vertex. Recall the definition of the affine function $d \phi\left(t_{i}\right)$. Let $g$ be the restriction of $d \phi\left(t_{i}\right)$ to the plane $z_{i}$, and let $V_{i} \subset V$ be the set of V-vertices which belong to the face $i$ of $P$.

Note that the correspondence $v \rightarrow z_{v}$, restricted to $V_{i}$, gives a realization of the abstract polygon which is the $i$ face of $P$ as a polygon that lies in the plane $z_{i}$ and whose vertices are the $z_{v}, v \in V_{i}$. Denote this realization by $P_{i}(z)$.

Consider now the case where $i$ is a dead vertex; i.e., all the labels near $i$ are 0 . Then $g$ is zero on all the vertices of $P_{i}(z)$, and, being affine, it follows that $g$ is zero on the plane $z_{i}$. So, in that case, $d \phi\left(t_{i}\right)$ and $\phi\left(z_{i}\right)$ are both zero on $z_{i}$, and, consequently, $d \phi\left(t_{i}\right)$ is a scalar multiple of $\phi\left(z_{i}\right)$. But the gradient of all the affine functions in $\phi\left(G r_{2}\right)$ is 1 and $d \phi\left(t_{i}\right) \in T_{\phi\left(z_{i}\right)} \phi\left(G r_{2}\right)$. Therefore $d \phi\left(t_{i}\right)=0$, and $t_{i}=0$. Conversely, it $t_{i}=0$, then obviously $d \phi\left(t_{i}\right)=0$ and $i$ is dead. Thus part (b) is proved in the case that $i$ is an F-vertex.

If $g$ is positive on all the vertices of $P_{i}(z)$, then all the labels near $i$ will be + , and there are no sign changes at $i$. Similarly, if it is negative on all these points, then all the labels near $i$ will be - . It remains to consider the case where $g$ is not all positive, all negative, or all zero on $z_{v}, v \in V_{i}$. Then there is a line, say $L$, contained in $z_{i}$ where $g$ is zero, and $g$ is positive on one half plane determined by this line and negative in the other. If the intersection of $L$ with $P_{i}(z)$ is an edge of $P_{i}(z)$, then two adjacent labels around $i$ are zero and the other labels are either all + or all - . (The labels which are 0 are those corresponding to the vertices of $P_{i}(z)$ which are in $L$.) Thus the total number of sign changes around $i$ is 2 in this case. Otherwise, $L$ intersects the 
boundary of $P_{i}(z)$ in at most two points. Every such intersection point corresponds to precisely one sign change in the total. (An intersection point in the relative interior of an edge of $P_{i}(z)$ corresponds to a sign change between a + and a - . An intersection point which occurs at a vertex $z_{w}$ of $P_{i}(z)$ corresponds to two half sign changes: each between the $l_{i, w}=0$ label corresponding to $w$ and the labels corresponding to its neighbors in the face $i$.) This completes the proof in the case $i \in F$.

Suppose now that $i \in V$. The proof for this case will be, in some sense, dual to the above. Assume, for convenience of notation, that $z_{i}=0$. We think of $t_{i}$ as a vector in $\mathbb{R}^{3}$. If $t_{i}=0$, then clearly all the labels near $i$ are 0 , and $i$ is dead. Conversely, if $l_{i, f}=0$ for all faces $f \in F$ incident with $i$, then $t_{i}$ belongs to all the corresponding planes $z_{f}$, and the intersection of these planes is $\left\{z_{i}\right\}$ $=\{0\}$, so $t_{i}=0$. Thus (b) is verified.

To prove (a), we may, and will, assume $t_{i} \neq 0$. Denote by $V_{i}$ the set of vertices $v \in V$ which neighbor with $i$, and denote by $C$ the convex hull of $P(z) \cup\left\{t_{i}\right\}$. Because $t_{i} \neq 0=z_{i}$, there are at most two faces of $C$ which contain $\left[t_{i}, z_{i}\right]$. Each face of $C$ which contains $\left[t_{i}, z_{i}\right]$ contains at most two points of the type $z_{v}$, $v \in V_{i}$. Let $v \in V_{i}$, and let $f, g \in F$ be the two faces which contain the edge $\langle i, v\rangle$. By the above, it is sufficient that we prove that the sum of the sign changes at $i$ in the triangles $\langle f, i, v\rangle$ and $\langle v, i, g\rangle$ is

(1) 0 , if $z_{v}$ does not belong to a face of $C$ which contains $\left[t_{i}, z_{i}\right]$,

(2) $\frac{1}{2}$, if $z_{v}$ belongs to only one face of $C$ containing $\left[t_{i}, z_{i}\right]$, and this face contains some other point of the form $z_{u}, u \in V_{i}$,

(3) at most 1 , in any case.

For the proof of (1), suppose that $z_{v}$ does not belong to a face of $C$ which contains $\left[t_{i}, z_{i}\right]$. Then the affine hull of $\left\{t_{i}, z_{i}, z_{v}\right\}$ must contain interior points of $P(z)$. Let $p=\lambda_{1} z_{v}+\lambda_{2} t_{i}$ be such an interior point. (Recall $z_{i}=0$.) $p$ is below $z_{f}$ and below $z_{g}$, but $0, z_{v} \in z_{f} \cap z_{g}$, and therefore $t_{i}$ is either below $z_{f}$ and below $z_{\mathrm{g}}$ (if $\lambda_{2}>0$ ), or above both of them (if $\lambda_{2}<0$ ). This implies that $l_{i, f}$ and $l_{i, g}$ are either both + or both - . Using Lemma 4.4, it follows that $l_{i, v}=l_{i, f}=l_{i, g}$, and we see that (1) holds.

To verify (2), assume now that $z_{v}$ and $z_{u}$ belong to a face, say $C_{0}$, of $C$, containing $\left[t_{i}, z_{i}\right], v \neq u \in V_{i}$, and that $C_{0}$ is the only face of $C$ containing $t_{i}$, $z_{v}, z_{i}$. The first assumption, implies that $u$ must belong to the face $f$ or to $g$, say to $f$. Therefore, because $C_{0}$ and $z_{f}$ both contain the points $z_{i}, z_{v}, z_{u}$, $C_{0}$ is contained in $z_{f}$. Thus $t_{i} \in z_{f}$, and $l_{i, f}=0$. On the other hand, the second assumption shows that $z_{g}$ does not contain a face of $C$ containing $\left[t_{i}, z_{i}\right]$. This gives $t_{i} \notin z_{\mathrm{g}}$, and therefore $l_{i, g} \neq 0$. Using this and $l_{i, f}=0$, Lemma 4.4 gives $l_{i, \mathrm{~g}}$ $=l_{i, v} \neq l_{i, f}=0$, and (2) follows.

(3) follows immediately from Lemma 4.4, and this completes the proof of the lemma.

4.7 Orientation Lemma. Let $\langle i, j\rangle$ be an edge in $G^{+}$, and suppose that $t \in T_{z} S_{\langle i, j\rangle}$. Then the labels $l_{i, j}$ and $l_{j, i}$ are complementary. That is, $l_{i, j}=+$ if $l_{j, i}=-, l_{i, j}=0$ if $l_{j, i}=0$, and $l_{i, j}=-$ if $l_{j, i}=+$.

If we suppose that $t \in \bigcap_{\langle i, j\rangle \in E \cup V F} T_{z} S_{\langle i, j\rangle}$, then the lemma implies that on every edge of $G^{+}$the labels are complementary, and so we can associate with $t$ a partial orientation of $G^{+}$: an edge is oriented from + to - , and the edges 
labeled with two 0's remain unoriented. This is the reason for calling this lemma the 'Orientation Lemma'.

Proof of Lemma 4.7 Consider the case where $\langle i, j\rangle$ is an edge of type V-V. Let $z(s)$ be a smooth curve in $S_{\langle i, j\rangle}$ satisfying $z(0)=z$ and $\left.\frac{d z(s)}{d s}\right|_{s=0}=t$. If $l_{i, j}=+$, the $t_{i}$ points above the plane $m_{\langle i, j\rangle}$, and for $s>0$ sufficiently small $z(s)_{i}$ will be above this plane. If also $l_{j, i}=+$, then $z(s)_{j}$ will be above that plane for $s>0$ sufficiently small. But this would mean that the line segment $\left[z(s)_{j}, z(s)_{i}\right]$ joining $z(s)_{j}$ and $z(s)_{i}$ lies above this plane, which contradicts $z(s) \in S_{\langle i, j\rangle}$, because $K$ does not intersect the half space above $m_{\langle i, j\rangle}$. Thus we see that two +labels cannot occur on the edge $\langle i, j\rangle$.

If $l_{i, j}=+, l_{j, i}=0$, then, for sufficiently small $s>0$, the segment $\left[z(s)_{j}, z(s)_{i}\right]$ is above $m_{\langle i, j\rangle}$, except possibly for points arbitrarily close to $z_{j}$. Again this gives a contradiction, because $K$ is disjoint from a neighborhood of $z_{j}$ and does not intersect the half space above $m_{\langle i, j\rangle}$. Thus we see that a + and a 0 are impossible.

Because $-t$ is also in $T_{z} S_{\langle i, j\rangle}$, we see that two - labels, or a - and a 0 are also impossible. The only remaining possibilities are + and - , or two 0 's, as required.

The proof for the case that $\langle i, j\rangle$ is a V-F type edge is similar (and simpler).

4.8 Lemma (Sign changes in a quadrilateral) Let $\langle v, u\rangle$ be a V-V type edge in $G^{+}$, and let the two faces of $P$ which contains this edge be $e, f \in F$. Suppose that $t \in T_{z} S_{\langle v, u\rangle} \cap T_{z} S_{\langle v, e\rangle} \cap T_{z} S_{\langle v, f\rangle} \cap T_{z} S_{\langle u, e\rangle} \cap T_{z} S_{\langle u, f\rangle}$. If among $v, u, e$, $f$ there is one live vertex, then the sum of the total number of sign changes in both triangles $\langle v, u, e\rangle$ and $\langle v, u, f\rangle$ of $G^{+}$is at least one. (See Definition 4.5.) If among these four vertices there is more than one live vertex, then this number of sign changes is at least two.

Proof. No new geometric arguments will appear in the proof - the lemma is a combinatorial consequence of the Orientation Lemma and Lemma 4.4. Consider some triangle $\langle i, j, k\rangle$ of $G^{+}$, and assume that not all the labels in it are 0 and that $t \in T_{z} S_{\langle i, j\rangle} \cap T_{z} S_{\langle j, k\rangle} \cap T_{z} S_{\langle k, i\rangle}$. We will show, by a case by case analysis, that the total number of sign changes in this triangle is at least one. Suppose, without loss of generality, that $l_{i, j} \neq 0$, and suppose, by symmetry, that $l_{i, j}=+$. Then $l_{j, i}=-$, by the Orientation Lemma. We check the three possibilities for the label $l_{i, k}$. If $l_{i, k}=-$, then we already have one full sign change in $\langle i, j, k\rangle$ at $i$. Consider the possibility $l_{i, k}=0$. Then there is half a sign change at $i$, and $l_{k, i}=0$, again by the Orientation Lemma. If $l_{k, j}=+,-$, then we also have half a sign change at $k$, and so only $l_{k, j}=0$ needs to be discussed. This however leads to $l_{j, k}=0$, and the half sign change appears at $j$. It remains to check $l_{i, k}=+$, and, by symmetry, only the possibility $l_{j, k}=-$ needs consideration. But then $l_{k, i}=-$ and $l_{k, j}=+$, giving a sign change at $k$.

We return to the situation of the lemma. Assume that the total number of sign changes in $\langle v, u, e\rangle$ and $\langle v, u, f\rangle$ is less than 2. By the above, it follows that in at least one of these triangles, say in $\langle v, u, e\rangle$, all the labels are 0 . In particular, we have $l_{v, u}=l_{v, e}=0$. From Lemma 4.4 it then follows that $l_{v, f}=0$ also, and by symmetry $l_{u, f}=0$. From the Orientation Lemma we 
get $l_{f, u}=l_{f, v}=0$, and so all the labels on both triangles are 0 . If, for instance, $v$ is live, than there are two half sign changes at $v$, one in each triangle. Thus there is a total of one sign change in our quadrilateral (the two neighboring triangles) at each live V-vertex. Also, there clearly is one sign change for each live F-vertex. This completes the proof of the lemma.

Proof of Theorem 4.1 Suppose now that $t$ is in the intersection (4.2). As we pointed out in the subsection devoted to the codimension count, it is enough to prove $t=0$. Consider the graph $G^{+}$with the labeling induced by $t$. Note that $t \in S_{\langle i, j\rangle}$ for every $\langle i, j\rangle \in E \cup V F$, and so Lemma 4.8 can be freely applied.

The basic idea is the oldest trick in the book of combinatorics - a double count. We will count the number of sign changes in two different ways. On the one hand, Lemma 4.6 tells us that there are 'few' sign changes, and on the other hand Lemma 4.8 shows that there are 'many' sign changes.

Each triangle of $G^{+}$has one V-V edge, and two V-F edges. We pair the triangles of $G^{+}$across V-V edges, to form quadrilaterals. Let $Q_{1}$ be the set of such quadrilaterals which have exactly one live vertex, and let $Q_{2}$ be the set of such quadrilaterals which have at least two live vertices. By Lemma 4.8 , the total number of sign changes in each quadrilateral in $Q_{n}$ is at least $n(n=1,2)$. So the total number of sign changes in $G^{+}$is at least $\left|Q_{1}\right|+2\left|Q_{2}\right|$. But Lemma 4.6 implies that the total number of sign changes in $G^{+}$is at most $2 l$, where $l$ is the number of live vertices in $G^{+}$. Thus we arrive at the inequality

$$
\left|Q_{1}\right|+2\left|Q_{2}\right| \leqslant 2 l \text {. }
$$

We think of this inequality as saying that there is a set of vertices $L$, the set of live vertices, which are incident to relatively few quadrilaterals; relative in relation to $l=|L|$. This is like having a set which is large when compared to the size of its boundary.

Our intention is to prove $l=0$. Then Lemma $4.6(\mathrm{~b})$ gives $t_{i}=0$ for each vertex $i$, and thus $t=0$, as required. There are fairly direct (traditional) ways to prove $l=0$ from inequality $(4.4)$, and from the fact that there is a triangle of dead vertices $-\left\langle v_{0}, v_{1}, f_{2}\right\rangle$. We prefer to take a shortcut.

Let $G^{\prime}$ be the planarly embedded graph obtained from $G^{+}$in the following manner. In each quadrilateral of $Q_{1}$ that has a V-type live vertex delete the $\mathrm{V}-\mathrm{V}$ edge, and insert into the quadrilateral an F-F edge joining the two F-type vertices of that quadrilateral. Because the graph $G^{\prime}$ is planar, $G^{\prime}$ can be embedded in the plane so that all its edges are straight line segments (see [Tu], or prove by induction on the number of vertices). Furthermore, there is such a straight embedding so that the unbounded region in the complement of the embedded $G^{\prime}$ is the region corresponding to the triangle $\left\langle v_{0}, v_{1}, f_{2}\right\rangle$, whose vertices are all dead. We think of $G^{\prime}$ as being thusly embedded.

We estimate in two different ways the sum of the angles of $G^{\prime}$ at the live vertices. On the one hand, this is clearly $2 \pi l$. To make another estimate of this quantity, let $q$ be some quadrilateral in $Q_{1}$, and let $i$ be the live vertex of $q$. By our construction of $G^{\prime}$, the angle of $q$ at $i$ is an interior angle of a triangle, and is thus smaller than $\pi$. Since the sum of the angles of any quadrilateral is $2 \pi$ (except for the quadrilateral containing $\left\langle v_{0}, v_{1}, f_{2}\right\rangle$ ), the sum of angles at live vertices for any quadrilateral in $Q_{2}$ is at most $2 \pi$, and is in fact smaller 
than $2 \pi$ unless all its vertices are live. Summing over all quadrilaterals the total of the angles at the live vertices we see that

$$
\pi\left|Q_{1}\right|+2 \pi\left|Q_{2}\right| \geqq 2 \pi l .
$$

Comparison with (4.4) shows that this inequality must be an equality. Thus our estimates for each quadrilateral are, in fact, realized. This shows that the vertices in every quadrilateral are either all dead, or all alive. By connectedness, since there are some dead vertices, all the vertices are dead. This completes the proof of Theorem 4.1 .

We now obtain corollaries from the Theorem.

4.9 Corollary (Rigidity) $Z_{P, K}^{\prime}$ is a discrete set.

Proof. Because (4.1) is a transverse intersection of $C^{1}$-smooth submanifolds, the intersection $Z_{P, K}^{\prime}$ is a $C^{1}$-smooth submanifold whose codimension is equal to the sum of the codimensions. By our codimension count, this means that $Z_{P, K}^{\prime}$ is a 0-dimensional submanifold; that is, it is discrete. 1.2.

The following elementary lemma will be needed for the proof of Theorem

4.10 Lemma. Let $X_{1}, X_{2}, \ldots, X_{n}, Y_{1}, Y_{2}, \ldots, Y_{m}, M$ be $C^{1}$ submanifolds of a $C^{1}$ manifold $Z$. Suppose that

$$
z \in\left(\cap_{i=1}^{n} X_{i}\right) \cap\left(\cap_{i=1}^{m} Y_{i}\right)
$$

and that intersection is transverse at $z$. Further suppose that

$$
M \supset \bigcap_{i=1}^{n} X_{i} .
$$

Then the intersection $M \cap\left(\cap_{i=1}^{m} Y_{i}\right)$ is transverse at $z$.

Proof. We will use the notation $\dot{X}_{i}$ for the tangent space of $X_{i}$ at $z, T_{z} X_{i}$. Similarly $\dot{Y}_{i}=T_{z} Y_{i}, \dot{M}=T_{z} M$. When $U \subset W$ are vector subspaces of $T_{z} Z$ we will denote the codimension of $U$ in $W$ by $\operatorname{codim}(U, W)$. The codimension of $U$ in $T_{z} Z$ is denoted by $\operatorname{codim}(U)$. In the following computation we use the fact that $\operatorname{codim}\left(\cap_{i} U_{i}, W\right) \leqq \sum_{i} \operatorname{codim}\left(U_{i}, W\right)$, and that $\operatorname{codim}(U)=\operatorname{codim}(U$, $W)+\operatorname{codim}(W)$, if $U, U_{i} \subset W$. Using the transversality of the intersection (4.5) and these properties, we have:

$$
\begin{aligned}
& \sum_{i=1}^{n} \operatorname{codim}\left(\dot{X}_{i}\right)+\sum_{i=1}^{m} \operatorname{codim}\left(\dot{Y}_{i}\right)+\operatorname{codim}(\dot{M}) \\
& \quad=\operatorname{codim}\left(\left(\cap_{i=1}^{n} \dot{X}_{i}\right) \cap\left(\cap_{i=1}^{m} \dot{Y}_{i}\right)\right)+\operatorname{codim}(\dot{M}) \\
& =\operatorname{codim}\left(\left(\cap_{i=1}^{n} \dot{X}_{i}\right) \cap\left(\cap_{i=1}^{m} \dot{Y}_{i}\right), \dot{M}\right)+2 \operatorname{codim}(\dot{M}) \\
& \leqq \operatorname{codim}\left(\cap_{i=1}^{n} \dot{X}_{i}, \dot{M}\right)+\operatorname{codim}\left(\cap_{i=1}^{m} \dot{Y}_{i} \cap \dot{M}, \dot{M}\right)+2 \operatorname{codim}(\dot{M}) \\
& =\operatorname{codim}\left(\cap_{i=1}^{n} \dot{X}_{i}\right)+\operatorname{codim}\left(\cap_{i=1}^{m} \dot{Y}_{i} \cap \dot{M}\right) \\
& \leqq \sum_{i=1}^{n} \operatorname{codim}\left(\dot{X}_{i}\right)+\sum_{i=1}^{m} \operatorname{codim}\left(\dot{Y}_{i}\right)+\operatorname{codim}(\dot{M}) .
\end{aligned}
$$


This show that equality holds throughout, and therefore $\operatorname{codim}\left(\cap_{i=1}^{m} \dot{Y}_{i} \cap \dot{M}\right)$ $=\operatorname{codim}(\dot{M})+\sum_{i=1}^{m} \operatorname{codim}\left(\dot{Y}_{i}\right)$. That is the required transversality at $z$, and the proof is complete.

Proof of Theorem 1.2 We do not yet prove the implicit claim that $Z_{P, K}$ is nonempty, but prove the rest. Consider some point $z \in Z_{P, K}$. For showing that $Z_{P, K}$ is a six dimensional submanifold near $z$ there is clearly no loss of generality in assuming that $z \in Z_{P, K}^{\prime}$, because of the freedom in the choice of $x_{0}, x_{1}, y_{2}$. Thus we will make this assumption.

Consider the set $M=Z_{+} \cap S_{\left\langle v_{0}, v_{1}\right\rangle} \cap S_{\left\langle v_{0}, f_{2}\right\rangle} \cap S_{\left\langle v_{1}, f_{2}\right\rangle}$. It is easy to see that $M$ is a $C^{k}$-smooth submanifold having codimension 3 , by showing that the intersection defining $M$ is transverse. (Recall that the $S_{\langle v, u\rangle}$ are $C^{k}$-smooth, by Lemma 3.1). In fact, the transversality of that intersection follows from Theorem 4.1 , because we could have chosen other vertices and another face in place of $v_{0}, v_{1}, f_{2}$.

We have

$$
Z_{P, K}=M \cap Z_{c} \cap\left(\cap_{\langle i, j\rangle \in E^{\prime} \cup V F^{\prime}} S_{\langle i, j\rangle}\right) .
$$

Since $M \supset Z^{0} \cap Z^{1} \cap Z^{2}$ and the intersection (4.1) is transverse, it follows from Lemma 4.10 that the intersection (4.6) is transverse at $z$. Therefore, in a neighborhood of $z, Z_{P, K}$ is a $C^{k}$-smooth submanifold whose codimension is equal to the sum of the codimensions in the intersection (4.6). So $\operatorname{codim}\left(Z_{P, K}\right)=3+\left|E^{\prime}\right|$ $+\left|V F^{\prime}\right|=\operatorname{codim}\left(Z_{P, K}^{\prime}\right)-6$, using our results from the codimension count subsection. Thus $\operatorname{dim}\left(Z_{P, K}\right)=6$, and this completes the proof.

Remark. It is probably true, and perhaps not too hard to prove, that the assumptions in Theorem 1.1 are sufficient to guarantee that $Z_{P, K}$ is a 6-manifold, perhaps not smooth.

\section{Existence of midscribing configurations}

In this section we will prove the Midscribability Theorem 1.1. The basic method is the following. We start with some realization of $P, z^{0} \in Z_{P}$, and show that there is a convex body, $K^{0}$, which has positively curved $C^{2}$-smooth boundary, and which $P\left(z^{0}\right)$ midscribes. Then we consider a curve of $C^{2}$-smooth convex bodies with positively curved boundary, $K^{s}, 0<s<1$, which joins $K^{0}$ to $K^{1}=K$. Take some $s \in[0,1)$, and assume that $K^{s}$ can be $P$-midscribed. It easily follows from Theorem 4.1 applied to $K^{s}$ that $K^{s^{\prime}}$ can be $P$-midscribed for all $s^{\prime} \in[0,1]$ sufficiently close to $s$. This shows that the set of $s \in[0,1)$ for which $K^{s}$ is $P$ midscribable is a relatively open set. If we could show that the set of $s \in[0,1]$ for which $K^{s}$ is $P$-midscribable is a closed set, then it would follow that this set is $[0,1]$, and that $K=K^{1}$ is $P$-midscribable. To show closure, the natural approach is to take a limit of midscribing configurations, and to claim that this gives a midscribing configuration. The difficulty in doing this is that the points $z_{v}$ may escape to infinity, or plunge into $K^{s}$. That is where the proof needs a little care, and where the details come in. 
The following lemma will enable us to make sure that the vertices stay within a compact subset of $\mathbb{R}^{3}$.

5.1 Lemma. For any smooth strictly convex body $K$ and for any polyhedron $Q$ midscribing $K$, there are at most two vertices of $Q$ whose distances from $K$ are greater than 2 diameter $(K)$.

Proof. Recall that if $p, q \in \mathbb{R}^{3}$ are two distinct vertices of $Q$, then the line segment joining $p$ and $q$ must intersect $K$. (Observation 2.1(2).) Now consider three distinct vertices of $Q, p, q, r \in \mathbb{R}^{3}$, and assume, without loss of generality, that $0 \in K$. If the angles between the vectors $p$ and $q$, and between the vectors $p$ and $r$ are $\geqq 2 \pi / 3$, then the angles between $-p$ and $q$, and between $-p$ and $r$ are $\leqq \pi / 3$. This would imply that the angle between $q$ and $r$ is $\leqq 2 \pi / 3$. Therefore, at least one of the three angles determined by $p, q, r$ at 0 is $\leqq 2 \pi / 3$. Assume that this is the angle between $p$ and $q$. Let $x$ be the point closest to 0 on the line segment joining $p$ and $q$. Because the angle between $p$ and $q$ is at most $2 \pi / 3$, it follows that $2\|x\| \geqq \operatorname{minimum}(\|q\|$, $\|p\|)$. But $\|x\| \leqq \operatorname{diameter}(K)$, because $0 \in K$, and $x$ is the closest point to 0 on the segment joining $p$ and $q$ which contains some point of $K$. Thus minimum $(\|q\|,\|p\|) \leqq 2 \operatorname{diameter}(K)$, and the lemma follows.

5.2 Lemma. Let $v_{0}, v_{1}$ be two neighboring vertices of $P$. For every constant $C>0$, there exists a realization of $P, z \in Z_{P}$, with the following property. The edge joining $z_{v_{0}}$ and $z_{v_{1}}$ has length $C$, but the distance from the midpoint of this edge to any vertex $z_{v}, v \neq v_{0}, v_{1}$ is less than $\frac{1}{2}$.

Proof. We start with an arbitrary realization $Q=P(z)$ of $P$, and modify $Q$ by a projective transformation. By applying an expansion, if necessary, assume that the edge $e=\left[z_{v_{0}}, z_{v_{1}}\right]$ has length $C$. Pick a plane $L_{0}$ in $\mathbb{R}^{3}$ which contains the edge $e$, but is otherwise disjoint from $Q$. Now think of $\mathbb{R}^{3}$ as being embedded in $\mathbb{R}^{4}$, and let $L \subset \mathbb{R}^{4}$ be an affine three space whose intersection with $\mathbb{R}^{3}$ is $L_{0}$. Let $x$ be the midpoint of the edge $e$, and let $o$ be a point in $\mathbb{R}^{4}-\left\{L \cup \mathbb{R}^{3}\right\}$ whose distance to $x$ is some small $\varepsilon>0$ and which is separated from $Q-e$ by $L$.

For any point $y \in \mathbb{R}^{3}$, if the line through $y$ and $o$ intersects $L$, then let $f(y)$ be the point in the intersection of this line with $L$. The mapping $f$ defined thusly is a projective map from $\mathbb{R}^{3}$ to $L$. (To be more precise, $f$ extends to a projective map from real projective 3-space to the projective closure of $L$.) The image of $Q, Q^{\prime}=f(Q)$, is a realization of $P$ in $L$. The points on the edge $e$ remain fixed under $f$.

On the other hand, let $h$ be the distance from $L$ to the vertices of $Q$ other than $z_{v_{0}}, z_{v_{1}}$, let $y$ be some vertex of $Q$ other than $z_{v_{0}}, z_{v_{1}}$, and let $l$ be the length of the part of $[y, o]$ which lies in the same side of $L$ as $y$. As Fig. 5.1 illustrates,

$$
d(o, f(y))=\frac{d(o, L) l}{d(y, L)} .
$$

Using $d(o, L) \leqq d(o, x)=\varepsilon, l \leqq d(y, o) \leqq d(y, x)+d(x, o)$, and $d(y, L) \geqq h$, we see that

$$
d(o, f(y)) \leqq \frac{\varepsilon(\text { diameter }(Q)+\varepsilon)}{h} .
$$

Thus, because the points $x, z_{v_{0}}, z_{v_{1}}$ remain fixed under $f$, the polyhedron $Q^{\prime}=f(Q)$ satisfys the required conditions, provided $\varepsilon$ is sufficiently small. 


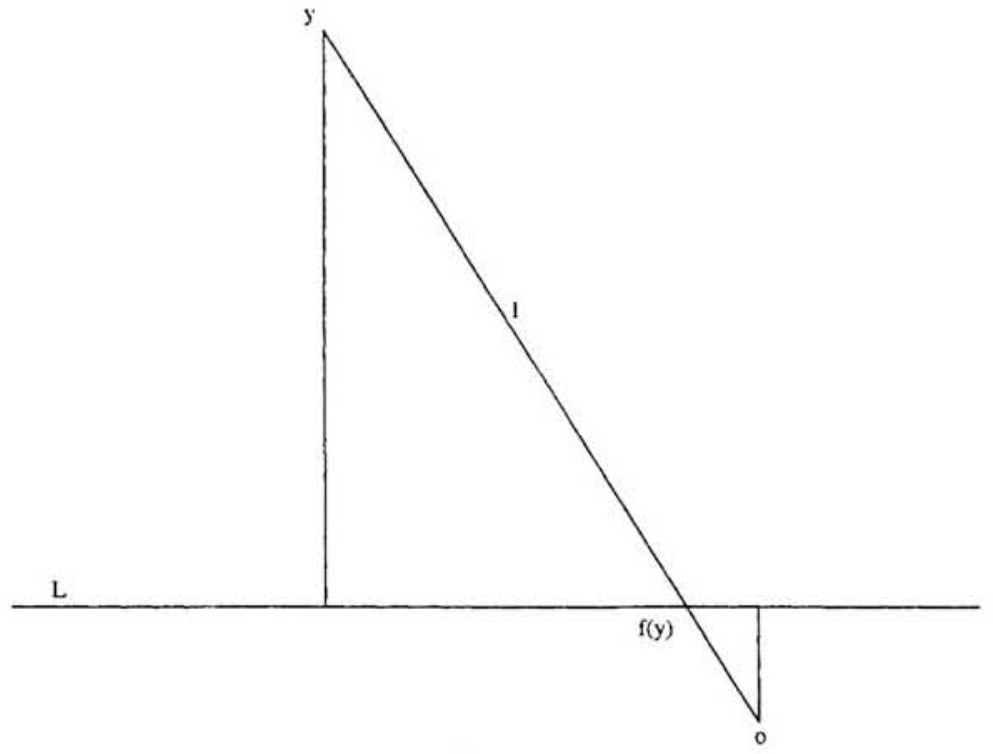

Fig. 5.1

Proof of Theorem 1.1 Let $v_{0}, v_{1}, f_{2}$ be as in Theorem 4.1. We start with a realization of $P, P\left(z^{0}\right), z^{0} \in Z_{P}$, which satisfies the conclusion of Lemma 5.2 with $C=6$. For each edge $e$ of $P\left(z^{0}\right)$, let $a_{e}$ be the midpoint of the edge. From the properties of $P\left(z^{0}\right)$ it follows that the diameter of the set of all such $a_{e}$ is $<1$. For each edge $e$ of $P\left(z^{0}\right)$ choose some plane $m_{e}$ which supports $P\left(z^{0}\right)$ at that edge; i.e., $P\left(z^{0}\right) \cap m_{e}=e$. Now let $K^{0}$ be a $C^{2}$-smooth strictly convex body of diameter $<1$ with positively curved boundary and whose boundary contains each $a_{e}$ and is tangent to $m_{e}$ at $a_{e}$ (for each edge $e$ of $P\left(z^{0}\right)$ ). Clearly, such a $K^{0}$ exists. (To get an explicit construction, one can start with the convex hull of small balls $B_{e}$, with each $B_{e}$ tangent to $m_{e}$ at $a_{e}$ from the side of $m_{e}$ which contains interior $\left(P\left(z^{0}\right)\right)$, and then minutely modify this convex body away from the $a_{e}$ to make its boundary positively curved and $C^{2}$ ). By construction, the polyhedron $P\left(z^{0}\right)$ midscribes $K^{0}$.

Denote by $x_{0}$ and $x_{1}$ the two vertices $z_{v_{0}}^{0}$ and $z_{v_{1}}^{0}$ of $P\left(z^{0}\right)$, let $e_{0}$ be the edge of $P\left(z^{0}\right)$ joining them, and let $y_{2}=z_{f_{2}}^{0}$. Let $K^{1}$ be a copy of $K$, rescaled and translated so that it has diameter $<1$ and is tangent to $m_{e_{0}}$ at $a_{e_{0}}$, from the same side that $K^{0}$ is. It is clearly enough if we show that $K^{1}$ can be $P$ midscribed.

Now choose a curve $K^{s}, s \in[0,1]$, of strictly convex bodies joining $K_{0}$ and $K_{1}$. We require that each of the sets $K^{s}, s \in(0,1)$, is tangent to $m_{e_{0}}$ at $a_{e_{0}}$, that its boundary be $C^{2}$-smooth and positively curved, and that its diameter be less than 1 (and the curve must be continuous, in the Hausdorff metric). Clearly, there exists such a curve.

Let $A$ be the set of $s \in[0,1]$ such that there exists a realization $z^{s} \in Z_{P}$ which midscribes $K^{s}$ and satisfys $z_{v_{0}}^{s}=x_{0}, z_{v_{1}}^{s}=x_{1}, z_{f_{2}}^{s}=y_{2}$. Clearly, $0 \in A$, because $P\left(z^{0}\right)$ midscribes $K^{0}$. Our goal is to show that $1 \in A$, by demonstrating that $A \cap[0,1)$ is relatively open and $A$ is closed in $[0,1]$.

Showing that $A \cap[0,1)$ is open in $[0,1]$ is easy after the preparations in the previous section: Consider an $s \in A-\{1\}$. The $K^{s}$ midscribing configuration 
$z^{s}$ is in $Z_{P, K^{s}}^{\prime}$. By Theorem 4.1, applied to $K^{s}$, the intersection (4.1) is transverse at $z^{s}$. This implies that if the intersecting submanifolds in (4.1) are perturbed slightly, an intersection close to $z^{s}$ remains. For $K^{\prime}$ a smooth strictly convex body sufficiently close to $K^{s}$, the corresponding submanifolds are close to those of $K^{s}$. (For each $\langle u, w\rangle \in E^{\prime}$ there is a homeomorphism of $S_{\langle u, w\rangle}\left(K^{s}\right)$ to $S_{\langle u, w\rangle}\left(K^{\prime}\right)$ which is arbitrarily close to the identity on compact subsets of $Z_{+}$, if $K^{\prime}$ is sufficiently close to $K^{s}$. For example, one can take as this homeomorphism the mapping $z \rightarrow z^{\prime}$ so that $z_{i}^{\prime}=z_{i}$ for $i \in F \cup V-\{u, w\}$, and $z_{u}^{\prime}=z_{u}+b^{\prime}-b$ and $z_{w}^{\prime}=z_{w}+b^{\prime}-b$, where $b$ is the point of tangency of $\left[z_{u}, z_{w}\right]$ with $K^{s}$ and $b^{\prime}$ is the point of $\partial K^{\prime}$ which has the same outer unit normal in $K^{\prime}$ as $p$ has in $K^{s}$. The submanifolds other than $Z_{+}$in the intersection (4.1) which are not of the form $S_{e}, e \in E^{\prime}$, are the same for $K$ and $K^{\prime}$.) Thus $Z_{P, K^{s^{\prime}}}^{\prime}$ is nonempty for $s^{\prime} \in[0,1]$ sufficiently close to $s$, proving that $A-\{1\}$ is open in $[0,1)$.

To prove that $A$ is closed in $[0,1]$, let $r_{1}, r_{2}, r_{3}, \ldots$ be a sequence in $A$ converging to some $r \in[0,1]$. Since $r_{n} \in A, n=1,2, \ldots$, there exists a configuration, $z^{r_{n}}$ say, in $Z_{P, K^{r_{n}}}^{\prime}$. The length of the edge $\left[x_{0}, x_{1}\right]$ is 6 , and the midpoint of this edge is in every $K^{s}$, and therefore, because diameter $\left(K^{s}\right)<1$, the distance from $x_{0}$ or $x_{1}$ to $K^{s}$ is greater than 2 diameter $\left(K^{s}\right)$. Together with Lemma 5.1, this implies that for every vertex $v \in V$ the sequence $\left\{z_{v}^{r_{n}}: n=1,2, \ldots\right\}$ is bounded. From this we also conclude that for every $f \in F$ the sequence $\left\{z_{f}^{r_{r}}: n=1,2, \ldots\right\}$ is contained in a compact subset of $G r_{2}$, because each plane $z_{f}^{r}$ contains some vertex $z_{v}^{r_{n}}$. Therefore there is some configuration $z \in Z$ which is an accumulation point for the sequence $\left\{z^{r_{n}}\right\}$. Since we can replace the sequence $\left\{r_{n}\right\}$ by some subsequence, we may, and will, assume that actually $z^{r_{n}} \rightarrow z$ as $n \rightarrow \infty$.

We want to show that $z$ is a midscribing configuration for $K^{r}$. It follows from the convergence of $z^{r_{n}}$ to $z$ and the corresponding property for $z^{r_{n}}$ that $z_{v}$ lies in the plane $z_{f}$, whenever the vertex $v \in V$ belongs to the face $f \in F$. Another consequence of the convergence is that for $v, u \in V\left[\mathrm{z}_{v}, \mathrm{z}_{u}\right]$ intersects $K^{r}$ (recall Observation 2.1(2)), and is tangent to $K^{r}$, if $\langle v, u\rangle \in E$. 'Tangent' here is taken in a broad sense - allowing the tangency to be at an endpoint of the segment $\left[z_{v}, z_{u}\right]$, or even allowing $z_{v}=z_{u} \in \partial K^{r}$. It still requires proof to show that $z_{v} \notin K^{r}$, $v \in V$.

To prove that $z_{v} \notin K^{r}, v \in V$, let $V_{0}$ be a connected component (in the graph of $P$ ) of the set of vertices $v \in V$ for which $z_{v} \in K^{r}$. Because $\left[z_{v}, z_{u}\right]$ is tangent to $K^{r}$ for $\langle v, u\rangle \in E$, it follows that all the points $z_{v}, v \in V_{0}$ are the same point, say $p_{0}$, and that $p_{0} \in \partial K^{r}$.

Let $f \in F$ be any face of $P$, and denote by $V_{f}$ the collection of vertices of $P$ which belong to $f$. Because $P\left(z^{r}\right)$ midscribes $K^{r_{n}}$ for each $n$, we know that the convex hull of $\left\{z_{v}^{r_{n}}: v \in V_{f}\right\}$ contains $K^{r_{n}} \cap z_{f}^{r_{n}}$ (Observation 2.1(1)). In the limit, since $K^{r}$ is strictly convex, it follows that the convex hull of $\left\{z_{v}: v \in V_{f}\right\}$ contains $K^{r} \cap z_{f}$.

Now, the face $z_{f_{2}}$ intersects the interior of $K^{r}$, since $z_{f_{2}}=z_{f_{2}}^{0}=y_{2}$, which is not equal to $m_{e_{0}}$, but passes through the point $a_{e_{0}}$ where $m_{e_{0}}$ and $K^{r}$ are tangent (because $z_{f_{2}} \supset\left\{x_{0}, x_{1}\right\}$ ). Using the above fact that the convex hull of $\left\{z_{v}: v \in V_{f_{2}}\right\}$ contains the two dimensional smooth set $K^{r} \cap z_{f_{2}}$, we see that at least three points in $\left\{z_{v}: z \in V_{f_{2}}\right\}$ are not in $K^{r}$. Therefore $V-V_{0}$ contains at least three vertices.

Now let $U_{0}$ be the set of vertices in $V-V_{0}$ which neighbor with some vertex in $V_{0}$. For each $v \in U_{0}$, the segment $\left[z_{v}, p_{0}\right]$ is tangent to $K^{r}$ at $p_{0}$. Therefore, all the points $z_{v}, v \in U_{0}$, lie in the plane, $m_{p_{0}}$ say, which is tangent to $K^{r}$ at 
$p_{0}$. Because $K^{r}$ is strictly convex, $p_{0}$ is the only point of $K^{r}$ in $m_{p_{0}}$. Therefore, if $u, v$ are distinct vertices in $U_{0}$, then $\left[\mathrm{z}_{u}, \mathrm{z}_{v}\right]$ must pass through $p_{0}$, since $\left[z_{u}, z_{v}\right]$ has to intersect $K^{r}$. This implies that there are at most 2 distinct vertices in $U_{0}$. But, as noted above, there are more than 2 vertices in $V-V_{0}$, and we see that $U_{0}$ is a set of at most two vertices which separates the graph of $P$. This contradicts the well known elementary fact that graphs of polyhedra are 3-connected (see [Gr, Theorem 11.3.1]), and this contradiction shows that $V_{0}=\emptyset$. Therefore $z_{v} \notin K^{r}$ for $v \in V$.

To show that $z \in Z_{P, K^{r}}^{\prime}$, all that remains to be seen is that $z \in Z_{c}$. For this, let $f \in F$ be a face of $P$, and let $w \in V$ be some vertex which does not belong to the face $f$. Because for each $n=1,2,3, \ldots, z_{w}^{r_{n}}$ is below the plane $z_{f}^{r_{n}}$, in the limit, $z_{w}$ is either on $z_{f}$, or below $z_{f}$. We have noted above that the convex hull of $\left\{z_{v}: v \in V_{f}\right\}$ must contain $z_{f} \cap K^{r}$. From this and $z_{w} \notin K^{r}$, we see that if $z_{w} \in z_{f}$, then the segment $\left[z_{w}, z_{v}\right]$ will be disjoint from $K^{r}$ for some $v \in V_{f}$, but we know that that is impossible. Therefore $z_{w}$ is below the plane $z_{f}$, and this establishes $z \in Z_{c}$.

Having shown that $z \in Z_{P, K^{r}}^{\prime}$, we conclude that $r \in A$. Thus $A$ is a closed set. Because $A-\{1\}$ is open and closed in $[0,1)$, and is nonempty $(0 \in A)$, it follows that $A-\{1\}=[0,1)$. Now $1 \in A$, because $A$ is closed in $[0,1]$. Therefore $K^{1}$, and also $K$, can be $P$-midscribed. This completes the proof.

\section{Concluding remarks}

As pointed out in the introduction, Thurston's proof of the midscribability of the sphere can be reversed, to give another proof of:

6.1 Corollary (Circle Packing Theorem) Let $G$ be a planar graph. Then there exists a circle packing on the sphere having $G$ as its tangency graph; that is, the circles of the packing are in one to one correspondence with the vertices of $G$ and two circles touch if and only if the corresponding vertices of $G$ share an edge.

Proof. It is easy to see that one can extend $G$ to a triangulation of $S^{2}$ by adding vertices and edges to $G$, but without adding an edge between two vertices which are already in $G$. Since it is sufficient to prove the claim for the extended graph, we may, and will, assume that $G$ is a triangulation. Then there is some polyhedron, say $Q$, whose graph is precisely $G$. Theorem 1.1 implies that there is such a polyhedron $Q$ which midscribes the unit sphere $S^{2} \subset \mathbb{R}^{3}$. For each vertex $v$ of $Q$, let $C_{v}$ be the set of points on $S^{2}$ which are visible from $v$; that is, the set of $p \in S^{2}$ so that the segment $[p, v]$ intersects $S^{2}$ only in $p$. Then the collection of these sets $C_{v}$ forms a circle packing on $S^{2}$ and two circles are tangent if and only if the corresponding vertices share an edge of $Q$.

Of course, our result here can be used to generalize the circle packing theorem to packings on the boundary of convex bodies. However, this already appears in [Schr].

Remark. Perhaps the best setting for the midscription problem is not in $\mathbb{R}^{3}$, but in $S^{3}$ ( $S^{3}$ considered as the double cover of projective 3-space, rather than as the one point compactification of $\mathbb{R}^{3}$ ). This is like allowing polyhedra obtained 
from convex polyhedra by projective transformations. One advantage to working in $S^{3}$ is that duality between vertices and faces, which did not appear in our discussion but lurked in the background, is more apparent. Another advantage is that there is no need to worry about vertices escaping to infinity, and so the Lemmas 5.1 and 5.2 can be deleted from the discussion. With the appropriate adjustment of the definitions and the situation in Theorem 4.1 to $S^{3}$, one gets that if the points $x_{0}, x_{1}$ are not antipodal, and the plane (that is, codimension 1 oriened great sphere) $y_{2}$ contains interior points of $K$, then $Z_{P . K}^{\prime} \neq \emptyset$. In fact, $Z_{P, K}^{\prime}$ consists of one single point. In this setting, it follows that the manifold of solutions is homeomorphic to the Möbius group.

\section{References}

[An1] Andreev, E.M.: On convex polyhedra in Lobačevskiĩ spaces. Mat. Sb., Nov. Ser. 81 (123), 445-478 (1970); English translation in Math. USSR, Sb. 10, $413-440$ (1970)

[An2] Andreev, E.M.: On convex polyhedra of finite volume in Lobačevskiir space. Mat. Sb., Nov. Ser. 83 (125), 256-260 (1970); English translation in Math. USSR, Sb. 12, 255-259 (1970)

[Ca] Cauchy, A.L.: Sur les polygones et polyèdres, second Mémoire. J. Éc. Polytechnique 19, 87-98 (1813)

[Eg] Eggleston, H.G.: Convexity. Cambridge: Cambridge University Press 1958

[Gr] Grünbaum, B.: Convex Polytopes. New York: Wiley 1967

[G-S] Grünbaum, B., Shephard, G.: Some problems on polyhedra. J. Geom. 29, 182-190 (1987)

[Koe] Koebe, P.: Kontaktprobleme der konformen Abbildung. Ber. Verh. Saechs. Akad. Wiss. Leipzig, Math.-Phys. Kl. 88, 141-164 (1936)

[Ro] Roth, B.: Rigid and flexible frameworks. Am. Math. Mon. 88, 6-21 (1981)

[Schr] Schramm, O.: Existence and uniqueness of packings with specified combinatorics. Isr. J. Math. (to appear)

[Schu] Schulte, E.: Analogues of Steinitz's theorem about non-inscribable polytopes. In: Böröczky, K., Tóth, G.F. (eds.) Intuitive geometry. Siófok, 1985 (Colloq. Math. Soc. János Bolyai, vol. 48, pp. 503-516). Amsterdam: North-Holland 1987

[St] Steinitz, E.: Über isoperimetrische Probleme bei konvexen Polyedern. J. Reine Angew. Math. 159, 133-143 (1928)

[Th] Thurston, W.P.: The geometry and topology of 3-manifolds. Princeton University Notes 1982

[Tu] Tutte, W.T.: How to draw a graph. Proc. Lond. Math. Soc. 52, 743-767 (1963) 